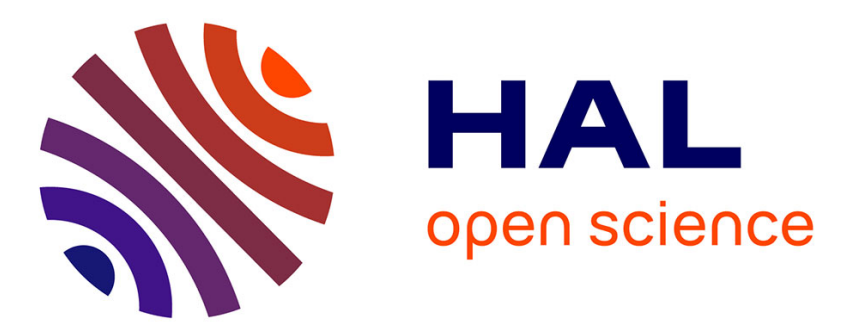

\title{
Improving user experience of SSVEP-BCI through reduction of stimuli amplitude depth
}

Simon Ladouce, Juan Jesus Torre Tresols, Ludovic Darmet, Giuseppe Ferraro, Frédéric Dehais

\section{> To cite this version:}

Simon Ladouce, Juan Jesus Torre Tresols, Ludovic Darmet, Giuseppe Ferraro, Frédéric Dehais. Improving user experience of SSVEP-BCI through reduction of stimuli amplitude depth. IEEE SMC 2021, IEEE, Oct 2021, Melbourne (virtual), Australia. hal-03388285

\section{HAL Id: hal-03388285 \\ https://hal.science/hal-03388285}

Submitted on 20 Oct 2021

HAL is a multi-disciplinary open access archive for the deposit and dissemination of scientific research documents, whether they are published or not. The documents may come from teaching and research institutions in France or abroad, or from public or private research centers.
L'archive ouverte pluridisciplinaire HAL, est destinée au dépôt et à la diffusion de documents scientifiques de niveau recherche, publiés ou non, émanant des établissements d'enseignement et de recherche français ou étrangers, des laboratoires publics ou privés. 


\title{
Improving user experience of SSVEP-BCI through reduction of stimuli amplitude depth
}

\author{
S. Ladouce ${ }^{1}$, J. J. Torre Tresols ${ }^{1}$, L. Darmet ${ }^{1}$, G. Ferraro ${ }^{1}$, and F. Dehais ${ }^{1,2}$
}

\begin{abstract}
Steady-States Visually Evoked Potentials (SSVEP) is currently one of the most widely used paradigms in Brain-Computer Interfaces (BCI). Although the SSVEP-BCI are characterized by their high and robust classification performance, the repetitive presentation of flickering stimuli is uncomfortable from a user experience perspective point of view. Indeed, the low-level visual features of SSVEP stimuli make them straining to the eyes over time and could be disruptive to the execution of tasks requiring sustained attention. They could even induce epileptic seizures. This study explores the reduction of the stimulation amplitude depth (a magnitude diminution of $\mathbf{9 0 \%}$ ) for the design of SSVEP stimuli as a solution to improve user comfort. The classification accuracy obtained by different pipelines was systematically compared between low amplitude and standard full amplitude SSVEP stimuli. The results reveal high classification accuracy for both high $(\mathbf{9 9 . 8 \%})$ and low magnitude $(80.2 \%)$ stimuli using the Task-Related Component Analysis (TRCA) classification method. The present findings demonstrate the validity of reducing SSVEP stimuli amplitude to increase users' comfort paving the way for transparent BCI operation.
\end{abstract}

\section{INTRODUCTION}

Steady-States Visually Evoked Potentials (SSVEP) refer to the sustained cortical entrainment to the frequency of periodical visual stimulations. When attending to repetitive visual stimuli (RVS) whose low-level features (color, luminance, shape) fluctuate at a certain rate (commonly through flickering shapes and alternating checkerboards), neuronal populations in the visual cortex synchronize their firing rate to the frequency of the visual stimulation [1]. The SSVEP is therefore well localized in the frequency domain of the electroencephalography (EEG) signal. Its signal in the EEG is most prominent at occipital sensor sites and source locations [2]. The SSVEP has been reported over a wide range of frequencies (1 to $80 \mathrm{~Hz}$, see [3]) which in the context of Brain-Computer Interfaces (BCI) offers the possibility to present an extended number of classes with discriminative signal features. Hence, SSVEPBCI are characterized by their high Information Transfer Rate (ITR) and classification accuracy. For instance, SSVEPBCI based systems using classification algorithms based on Task-Related Component Analysis (TRCA) reported very high ITR (over 200 bits/minute) for a 40-target application (i.e., keyboard) thus offering a wide range of interactive commands [4]. Furthermore, the minimal training (e.g., using calibration-free classification methods based on Canonical

\footnotetext{
1 ISAE-SUPAERO, Université Fédérale de Toulouse, France. 2 School of Biomedical Engineering, Science Health Systems, Drexel University, Philadelphia, PA, United States simon. ladoucedisae-supaero.fr
}

Correlational Analysis (CCA), [5]) required by some of the SSVEP-BCI methods further established SSVEP paradigms as a reliable and efficient approach for BCI applications.

Nonetheless, current SSVEP-based BCIs have several pitfalls. Like many other paradigms used in BCI, SSVEP requires the user to direct his attention and gaze toward visual stimuli related to the command that corresponds to his intention. This constraint usually results in back-and-forth eye movements, and therefore divided attention, between the interface where the stimuli are displayed and the actual object affected by the commands. Another important concern regarding SSVEP-based BCI is the ocular fatigue induced by the prolonged presentation of flickering visual stimuli [6]. The strength (i.e., magnitude in terms of luminance/contrast modulations) of the visual flickers is positively correlated with the induced SSVEPs amplitude response [7]. As a result, SSVEP paradigm designs have generally focused on maximizing SNR through the presentation of very salient, high-energy, visual stimuli. Furthermore, the amplitude of the SSVEP was found to be maximal when the stimuli flickers at a relatively low rate (ranging from 8 to $18 \mathrm{~Hz}$ ) [8] [9]. The repetitive presentation of visual information within such a low/intermediate frequency range is particularly straining on the eyes [6] and has been reportedly deemed annoying/frustrating by the users [10]. Another important consideration regarding the flickering frequency lies in the risk of epileptic seizures. Frequencies ranging from 4 to $20 \mathrm{~Hz}$ present acute risks of triggering epileptic seizures [11] [12]. According to the prevalent theory of a limited pool of cognitive resources [13], sensory information whose lowlevel features are particularly salient drive bottom-up (i.e., object-oriented as the SSVEP stimuli) sensory and attentional processing at the expense of top-down (i.e., cognitive driven) processing strategies. In contexts where cognitive resources need to be allocated to attend to different aspects of a task, SSVEP stimuli may grasp attention away from the actual task at hand. Introducing such interference may be detrimental to the performance of the task. This is in clear opposition to the very aim of $\mathrm{BCI}$ applications directed at supporting individuals in the execution.

In order to make the SSVEP-based BCI accessible to a wide population and increase its comfort of usage, it is crucial to reduce the intrusiveness of visual stimulations. This study explores the reduction of stimulation amplitude depth for the design of SSVEP-based BCI applications as a potential solution to the aforementioned issues. To meet this goal, we compared classic flickers (100\% magnitude depth) and low-amplitude (10\% magnitude depth) flickers 
in terms of subjective rating, signal-to-noise ratio, and classification accuracy using two classification pipelines namely CCA, TRCA. Decreasing the stimulation amplitude depth reduces the contrast between the two alternating states of the stimuli (ranging from grey to white for the control high amplitude condition and ranging from grey to lighter grey for the low-amplitude condition) but also reduces their maximal luminance (high amplitude stimuli have a maximum luminance 10 times higher than low amplitude stimuli).

\section{MAterial AND Method}

\section{A. Participants}

Eight healthy volunteers ( 2 women, median age: 28 years, range: 24-29 years) with normal or correctedto-normal vision took part in this study. Participant 2 withdrew his participation reporting a high level of discomfort following the presentation of high amplitude stimuli (epilepsy screening questionnaire was negative). As a result, an additional participant (Subject 9) was recruited as a replacement to ensure a proper counterbalancing of experimental blocks presentation order. The participants did not report any of the exclusion criteria (neurological antecedents, being under psychoactive medication at the time of the study). The study was approved by the ethics committee of the University of Toulouse (CER approval number 2020-334) and was carried in accordance with the declaration of Helsinki. Participants gave informed written consent prior to the experiment.

\section{B. Apparatus and stimuli}

Visual rhythmic stimuli were presented on an LCD monitor (LG GN750 27-inch, 1ms IPS display, NVIDIA G-sync compatible), with a refresh rate of 240 frames per second, a resolution of $1024 \times 768$ pixel (width $\mathrm{x}$ height) and a luminance of $400 \mathrm{~cd} / \mathrm{m} 2$. The visual rhythmic stimuli consisted in the presentation of series of squares (side of 200 pixels) at the centre of the screen whose colour state was alternating between grey and white at specific frequencies following a square function (see Figure 1). The choice of stimulus frequencies for the different classes was based upon 1) avoiding using a lower range of frequency to reduce eye strain [10] [6], 2) avoiding endogenous activity found in the alpha range $(8-12 \mathrm{~Hz}), 3)$ the restrictions relative to the refresh rate of the monitor (temporal resolution is limited by the integer number of frames that can be drawn every period). As a result, the following frequencies were selected: 13.33, $17.14,21.81$ and $24 \mathrm{~Hz}$. Each stimulus type (4 frequencies x 2 different magnitude depths) was presented 30 times for a total of 240 trials spread over four randomized blocks of 60 trials. Every trial lasted 6 seconds (4 seconds of stimulus presentation +2 seconds of interstimulus interval, see Figure 1), therefore the experiment itself lasted for approximately 25 minutes including short breaks between blocks. The visual stimuli were generated using Psychopy functions [14] running on Python 3.7. The visual flickers were presented against a grey background.

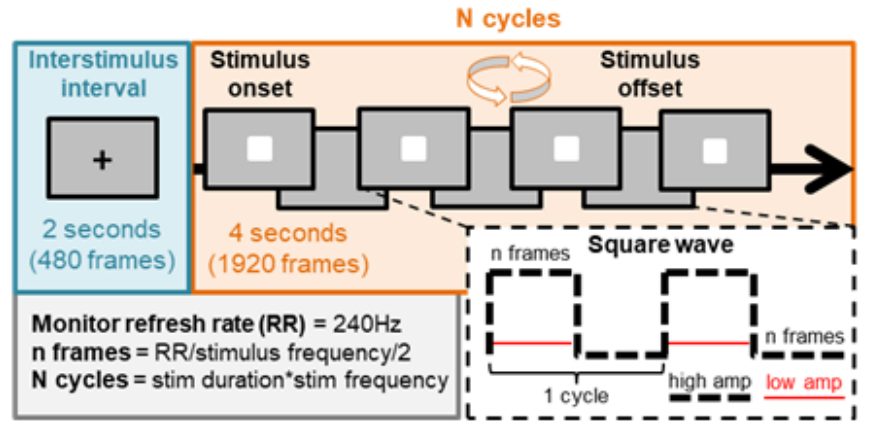

Fig. 1: Schematic representation of a single-trial SSVEP stimulus. Each trial is preceded by a 2 seconds (or 480 frames) interstimulus interval period during which a fixation cross was presented at the center of the screen. SSVEP stimuli are then presented for 4 seconds (or a total of 1920 frames). The SSVEP-eliciting stimuli is in practice the variation of transparency amplitude for a square presented at the center of the screen. The transparency follows a square waveform with a 50-50 duty cycle. The magnitude of the low amplitude flickers (red line) is set to be 10 times lower than in the high amplitude condition.

\section{Experimental design and procedure}

A four-class problem was used as a benchmark to compare the classification performance of low and highamplitude SSVEP stimuli. Participants were instructed to look at the centre of the screen where fixation crosses and stimuli were presented. Prior to the experiment, a series of visual stimuli flickering at the four different frequencies for both experimental conditions used in the experiment were presented to the participants to assess whether they experience eye strain or any discomfort related to the viewing of such stimuli by filling a self-reported questionnaire (stimuli were presented for 4 seconds after which participants filled-in the questionnaire, stimuli presentation order was randomized). The participants rated each stimulus on two Likert scales (ranging from 0 to 10) based on their subjective perception of how strongly the stimuli flickered and how comfortable the viewing of these stimuli was. The participants were then equipped with the EEG system. The capping procedure took from 20 to 30 minutes depending on the subject. As mentioned above, experimental blocks lasted for 25 minutes on average (including breaks between blocks) which amounts to a total participation time that did not exceed one hour.

\section{EEG recording}

EEG data were recorded from 32 active (Ag/AgCl) electrodes fitted in an elastic cap according to the 1020 international system and connected to a LiveAmp amplifier (Brain Products, Munich, Germany). The ground electrode was placed at the $\mathrm{Fpz}$ electrode location and all electrodes were referenced to the $\mathrm{FCz}$ electrode. The electrode impedance was brought below $20 \mathrm{k} \Omega$ prior to recording. The data were acquired at a rate of $500 \mathrm{~Hz}$ with 
an online digital band-pass filter ranging from 0.1 to $250 \mathrm{~Hz}$. At the onset of every stimulus presentation, an event trigger was generated by the stimulus presentation program and synchronized to the EEG data stream with Lab Streaming Layer (LSL) 1

\section{E. EEG Processing and Analyses}

The raw continuous EEG data have undergone an offline, high-pass filtering at $1 \mathrm{~Hz}$ (zero phase, acausal, filter order: $1651,-6 \mathrm{~dB})$ and re-referenced to the average of all channels. Continuous EEG data were then epoched around event timestamps ( -2 to 4 seconds epochs with flicker onset as time point 0 ). No channel was removed nor interpolated. Analyses were then carried out on the 4 seconds following stimulus onset. This study first characterized the magnitude of the SSVEP elicited for each frequency for both low and high amplitude conditions using Rhythmic Entrainment Source Separation (RESS, [15]). This comparison was performed on Signal-to-Noise Ratio (SNR) measures computed at RESS component level as recommended by [15]. RESS first computes channel-to-channel covariance matrices from narrow-band filtered data at stimulation frequencies (using a gaussian-shape filter of full width half maximum (FWHM) $=.5 \mathrm{~Hz}$ ) and neighbouring frequencies ( $\mathrm{R}$ matrices) (distance $=1 \mathrm{~Hz}$, neighbour filters FWHM $=1 \mathrm{~Hz}$ ). A generalized eigendecomposition is computed between the frequency stimulation and the average of neighbouring frequencies covariance matrices. The eigenvector with the largest eigenvalue is selected as the main RESS component. This latter component is then back-projected (acting as a spatial pattern) to the time series EEG data to maximize the SNR of SSVEP responses.

\section{F. BCI Analyses}

In order to assess the viability of using low amplitude flickering stimuli, the performance of two state-of-the-art classification pipelines were contrasted: one based on CCA and TRCA.

\section{1) Canonical Correlation Analysis (CCA)}

In recent years, $\mathrm{CCA}$ has become the gold standard method for the detection of frequency information in SSVEPbased BCI [5], [16]. Indeed, CCA-based methods have demonstrated high classification performance (ITR of 267 bits/min for an online 40-target SSVEP speller [17]). One of the main advantages of the standard CCA is that it does not require any training or calibration data. Formally, it can be formulated as follows: let be $\mathbf{X}$ and $\mathbf{Y}$ two multivariate random variables. CCA looks for two weight vectors $w_{x}$ and $w_{y}$ that maximize the correlation between $w_{X}^{T} \mathbf{X}$ and $w_{y}^{T} \mathbf{Y}$ :

$$
\underset{\left(w_{x}, w_{y}\right)}{\arg \max } \operatorname{corr}\left(w_{x}^{T} \mathbf{X}, w_{y}^{T} \mathbf{Y}\right)
$$

This maximal correlation is the so-called canonical correlation.

In our context, $\mathbf{X} \in \mathbb{R}^{N_{c} \times N_{s}}$ corresponds to a matrix of $N_{c}$ EEG channels by $N_{s}$ samples for a given epoch of data

\footnotetext{
${ }^{1}$ https://github.com/scen/labstreaminglayer
}

and $\mathbf{Y}_{f} \in \mathbb{R}^{N_{w} \times N_{s}}$ corresponds to a matrix with the same length of $X$ composed by $N_{w}$ artificial sine-cosine waves for the target frequency $f$, as well as their first harmonic. Then, one looks for the frequency $f$ of the artificial sine-cosine waves $\mathbf{Y}_{f}$ that maximizes the canonical correlation with our signal of interest $\mathbf{X}$. We followed recommendations from [16] regarding channel selection $(\mathrm{O} 1, \mathrm{Oz}, \mathrm{O} 2, \mathrm{P} 3, \mathrm{Pz}, \mathrm{P} 4)$ and the number of harmonics (1) of the artificial sin-cosine waves to consider.

\section{2) Task-Related Component Analysis (TRCA)}

The second method, TRCA [18] has been recently applied to SSVEP-BCI data recorded with both wet [4] and dry [19] EEG systems with remarkable results. The method has achieved around $90 \%$ of classification accuracy for 12 and 40 classes. While this approach requires the use of calibration data, it provides substantial improvements over other classification methods. For instance, [4] achieved an ITR value of $325.33 \mathrm{bits} / \mathrm{min}$ using a dry electrodes system. The TRCA method takes advantage of the filter bank analysis as described in [4], with the same parameters. Thus the following steps are repeated across the $m$ bands obtained with the filter bank. In this study $m=5$ sub-bands were set to $m \times 13$ and $90 \mathrm{~Hz}$, following [4].

\section{Filtering}

First, the method uses calibration data to generate spatial pattern (a filter combining EEG channels) $w_{f}^{(m)}$ for each class.

The spatial pattern for frequency $f$ is obtained by solving the following optimization problem:

$$
\widehat{w_{f}^{(m)}}=\underset{w}{\arg \max } \frac{w^{T} \mathbf{S} w}{w^{T} \mathbf{Q} w}
$$

Which can be solved by finding the leading eigenvector of the matrix $\mathbf{Q}^{-1} S$, where $\mathbf{Q}$ is the covariance $\left(\in \mathbb{R}^{N_{c} \times N_{c}}\right)$ of the concatenated matrix of all trials with a stimulation frequency $f$ and $\mathbf{S}$ is the sum of all covariances $\left(\in \mathbb{R}^{N_{c} \times N_{c}}\right)$ between separate trials for that same stimulation frequency. $w^{T} \mathbf{S} w$ is the inter-trials covariance of filtered data. $w^{T} \mathbf{Q} w$ is the variance of the filtered data, it need to be bounded to obtained a finite solution for $\widehat{w_{f}^{(m)}}$.

\section{Individual templates construction}

The individual data templates $\overline{\mathbf{X}}_{f}^{(m)} 1, \overline{\mathbf{X}}_{f}^{(m)} 2, \ldots, \overline{\mathbf{X}}_{f}^{(m)} N$ are obtained by averaging all the calibration data epochs (on the seconds following stimulus onset) for the corresponding frequency (i.e., class) and band of the filter bank. These calibration data are then discarded and not used for performance computation. After the calibration, any new data observation is divided into $m$ sub-bands, and then the correlation coefficient between $w^{T(m)} \mathbf{X}^{(m)}$ and $w^{T(m)} \overline{\mathbf{X}}^{(m)}$ is computed. The correlation scores for each sub-band are combined using a weighted sum (see [19] for more details). After repeating the process for all $N$ frequencies, the maximal value across the $N$ correlation coefficients is interpreted as the estimated frequency of the trial. 


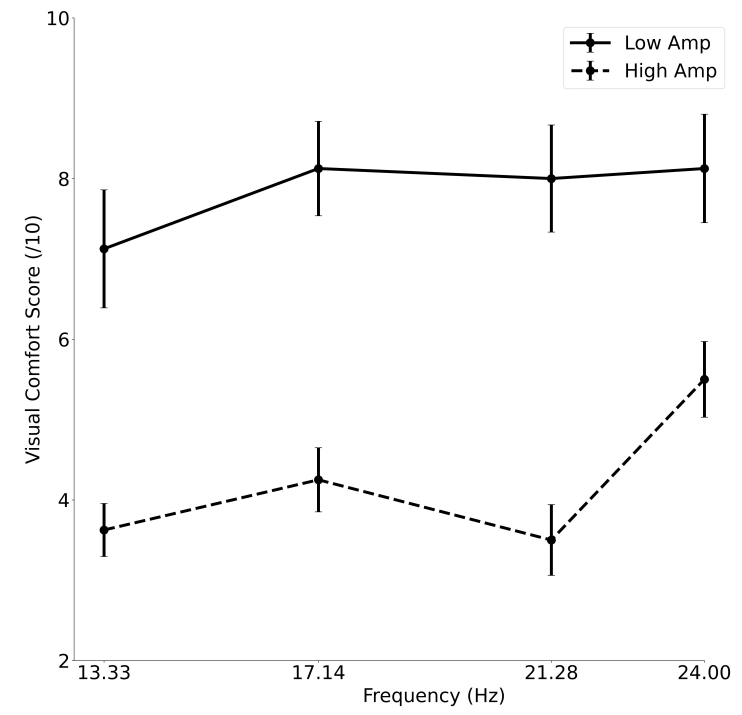

Fig. 2: Distribution of mean visual comfort score for both amplitude modulation conditions (low and high) and each stimulus frequency $(13.33,17.14,21.81,24 \mathrm{~Hz})$

\section{RESULTS}

\section{A. Subjective stimulus assessment}

A $2 \times 4$ repeated measures ANOVA was performed to investigate the effects of stimuli amplitude ( 2 conditions: low and high) and frequency (4 conditions: 13.33, 17.14, $21.81,24 \mathrm{~Hz}$ ) on the perceived strength of the visual flickers and how comfortable the visual flickers were rated by the participants.

For the perceived strength of the visual flickers, no main effect of the amplitude variation $[\mathrm{F}(1,7)=1.800, \mathrm{p}=0.222$, $\left.\eta^{2}=.048\right]$ nor the stimulus frequency $[\mathrm{F}(1,7)=0.748, \mathrm{p}=$ $\left.0.536, \eta^{2}=.039\right]$. No interaction between stimulus amplitude reduction and frequency was found for the subjective report of stimulus strength $\left[\mathrm{F}(3,21)=1.655, \mathrm{p}=0.207, \eta^{2}=0.069\right]$.

The subjective rating of stimuli comfort was mainly affected by both amplitude reduction $[\mathrm{F}(1,7)=93.444$, $\mathrm{p}$ $\left.<.001, \eta^{2}=0.752\right]$ and the stimulus frequency $[\mathrm{F}(1,7)$ $\left.=9.035, \mathrm{p}<.001, \eta^{2}=0.066\right]$. Moreover an interaction between the amplitude and the frequency of the stimulus was found to affect how comfortable the stimuli were rated by the participants $\left[\mathrm{F}(3,21)=3.789, \mathrm{p}=0.026, \eta^{2}=0.026\right]$. Posthoc t-tests (with a Holm correction applied) revealed that the low amplitude stimuli were rated as more comfortable (mean difference $=3.625$ ) than the high amplitude stimuli [p $<.001, \mathrm{~d}=3.418$ ]. Moreover, stimuli at higher frequencies were rated as more comfortable than stimulus flickering at lower frequencies (see Figure 2).

\section{B. SSVEP SNR}

The SSVEP SNR of the main RESS component for each stimulus frequency across both amplitude modulation conditions (low and high) are presented in Table II A $2 \times 4$ repeated measures ANOVA was performed to investigate the effects of stimulus amplitude (low and high) and frequency

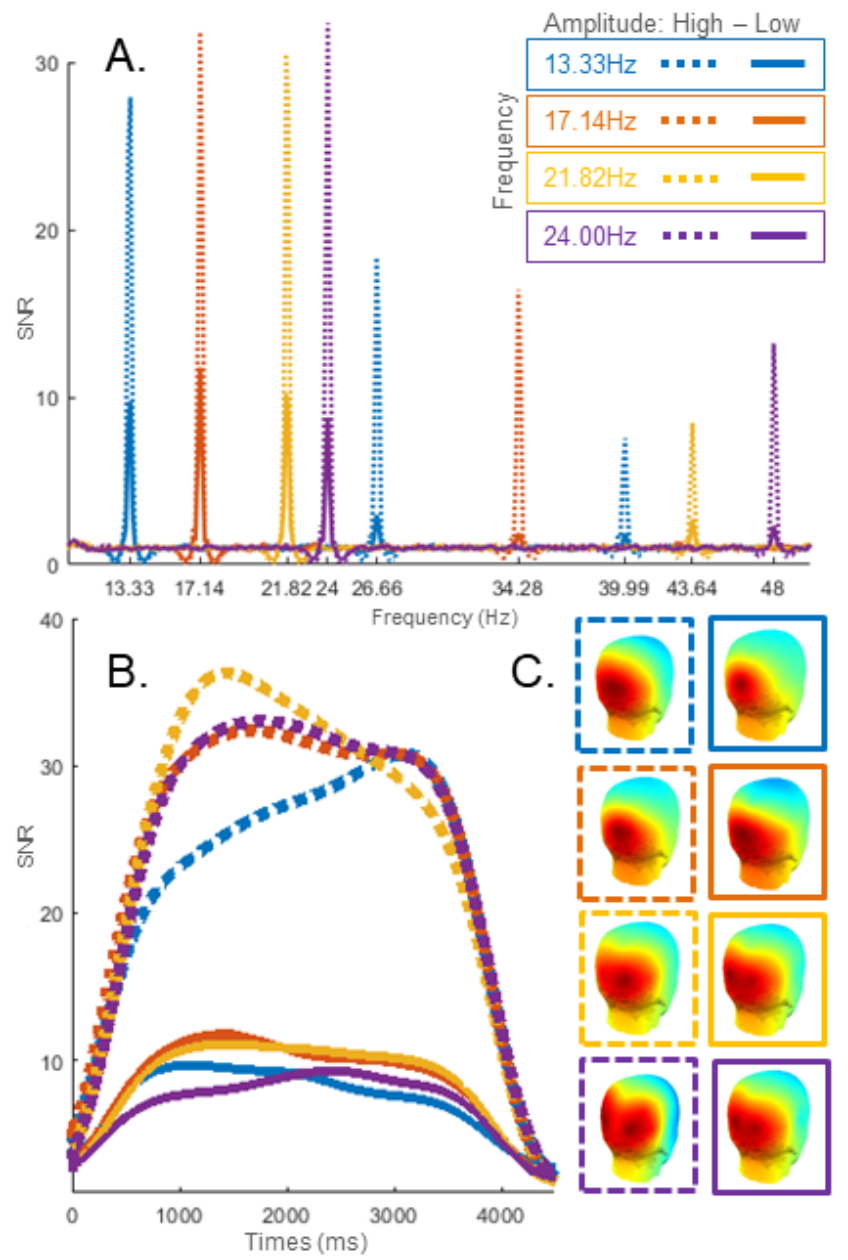

Fig. 3: Grand average SSVEP Signal-to-Noise Ratio (SNR). A) Power spectrum of the different stimulus frequencies $(13.33 \mathrm{~Hz}$ - blue, $17.14 \mathrm{~Hz}$ - orange, $21.81 \mathrm{~Hz}$ - yellow, $24 \mathrm{~Hz}$ - purple) for both amplitude modulation conditions (high dotted line, low - solid line). B) Mean time-course of SSVEP SNR over the length of stimulus presentation period. C) Topographical distribution of the RESS main component.

$(13,17,21,24 \mathrm{~Hz})$ on the SSVEP SNR of the main RESS component.

A main effect of the amplitude reduction $[\mathrm{F}(1,7)=17.421$, $\left.\mathrm{p}=0.004, \eta^{2}=.587\right]$ was observed on SSVEP SNR. In contrast, neither the stimulus frequency $[\mathrm{F}(3,21)=0.611$, $\mathrm{p}$ $\left.=0.615, \eta^{2}=.006\right]$ nor the interaction between stimulus amplitude reduction and frequency $[\mathrm{F}(1.4,9.6)=0.383, \mathrm{p}$ $\left.=0.616, \eta^{2}=0.005\right]$ did not yield a significant effect on SSVEP SNR. Greenhouse-Geisser correction was applied when the sphericity assumption was violated. Post-hoc paired sample t-tests (with a Holm correction applied) revealed that low amplitude stimuli had a significantly lower SNR (mean difference $=20.5$ ) than the high amplitude stimuli $[\mathrm{p}=.004$, $\mathrm{d}=1.476$ ], see Figure 3 . 


\begin{tabular}{lllllllll} 
& \multicolumn{3}{c}{$13.33 \mathrm{~Hz}$} & \multicolumn{2}{c}{$17.14 \mathrm{~Hz}$} & \multicolumn{2}{c}{$21.81 \mathrm{~Hz}$} & \multicolumn{2}{c}{$24 \mathrm{~Hz}$} \\
\cline { 2 - 9 } & High & Low & High & Low & High & Low & High & Low \\
\hline Subject 1 & 20.1 & 3.5 & 14.7 & 5.5 & 11.3 & 4.6 & 18.4 & 3.5 \\
Subject 3 & 32.1 & 3.3 & 40.8 & 3.9 & 53 & 7.6 & 48.5 & 8 \\
Subject 4 & 25.3 & 16 & 33.2 & 18.2 & 34.1 & 10.2 & 44.4 & 7.5 \\
Subject 5 & 18.7 & 7.7 & 27.6 & 9.4 & 19.2 & 6.4 & 30.4 & 6.9 \\
Subject 6 & 13 & 17.3 & 19.4 & 18 & 54.5 & 39.9 & 30.4 & 18.8 \\
Subject 7 & 15.3 & 18 & 20.2 & 17.3 & 15.5 & 11.4 & 24.1 & 25.4 \\
Subject 8 & 6.3 & 5.1 & 6.2 & 3.6 & 18.4 & 25.1 & 14.8 & 14 \\
Subject 9 & 12.9 & 16.8 & 7 & 5.5 & 38.7 & 61.9 & 57 & 58.7 \\
\hline Mean & 27.9 & 9.8 & 31.8 & 11.8 & 30.5 & 10.2 & 32.3 & 8.8 \\
\pm std & 13.3 & 5.2 & 16.2 & 6.4 & 16.9 & 6.1 & 16.4 & 5.7
\end{tabular}

TABLE I: Main RESS component Signal-to-Noise Ratio (SNR) over the whole 4 seconds epochs for each stimulation frequency $(13.33,17.14,21.81,24 \mathrm{~Hz})$ and amplitude modulation (high and low).

\section{BCI results}

The results of the offline analyses performed on the whole stimulus presentation period are listed in Table III For the high amplitude condition, the mean accuracy across all subjects reached $98.2 \% \pm 2.1$ with the CCA method and $99.8 \% \pm 0.6$ with TRCA. For the low amplitude condition, a mean accuracy of $62.8 \% \pm 20.7$ and $80.2 \% \pm 15.8$ were performed by the CCA and TRCA methods respectively. The minimal accuracy was observed in the low amplitude condition for subjects 1,8 and 9 .

Figure 4 shows the accuracy of CCA and TRCA methods over different data lengths $(0.5,1,2,34$ seconds). It can be observed that the TRCA method not only achieves higher classification accuracy for both the high and low amplitude conditions but also that the variance across subjects is also lower than CCA. Importantly, TRCA requires shorter data lengths than CCA to reach high classification accuracy and CCA performance continues to improve with longer data lengths whereas TRCA shows diminishing returns after 2 and 3 seconds of data for the high and low amplitude conditions. This result is of particular interest for the design of responsive BCI that operates on short time series. Overall, the TRCA method showed higher classification performance than the standard CCA approach for both high $[\mathrm{t}(7)=2.531$, $\mathrm{p}=0.039, \mathrm{~d}=.895]$ and low amplitude $[\mathrm{t}(7)=3.151, \mathrm{p}=$ $0.016, \mathrm{~d}=1.114]$ stimuli.

\section{Discussion}

The main motivation of this study was to assess the design of low amplitude SSVEP-based stimuli to improve user comfort and as a first step toward the implementation of transparent BCI. To this end, we have designed an experiment to compare SSVEP stimuli, presented at four different frequencies, under two magnitude levels: high $(100 \%)$ and low (10\%). As expected, the subjective rating scores revealed that low amplitude stimuli were perceived as more comfortable than the high amplitude stimuli. It is worth noting that higher frequencies were also deemed as more comfortable than lower frequencies. These results further suggest that decreasing the intensity but also increasing

\begin{tabular}{lllll} 
& \multicolumn{2}{c}{ CCA } & \multicolumn{2}{c}{ TRCA } \\
\cline { 2 - 5 } & High & Low & High & Low \\
\hline Subject 1 & 97.5 & 60 & 100 & 67.5 \\
Subject 3 & 100 & 56.7 & 100 & 78.3 \\
Subject 4 & 96.6 & 51.7 & 100 & 84.2 \\
Subject 5 & 100 & 45.8 & 100 & 82.5 \\
Subject 6 & 100 & 99.2 & 100 & 100 \\
Subject 7 & 97.5 & 90.8 & 100 & 100 \\
Subject 8 & 94.2 & 55 & 98.3 & 52.5 \\
Subject 9 & 100 & 43.7 & 100 & 76.7 \\
\hline Mean & 98.2 & 62.8 & 99.8 & 80.2 \\
\pm std & 2.1 & 20.7 & 0.6 & 15.8
\end{tabular}

TABLE II: Comparison of classification accuracy obtained using the Canonical Correlation Analysis (CCA) and TaskRelated Component Analysis (TRCA) methods for both amplitude modulation conditions.

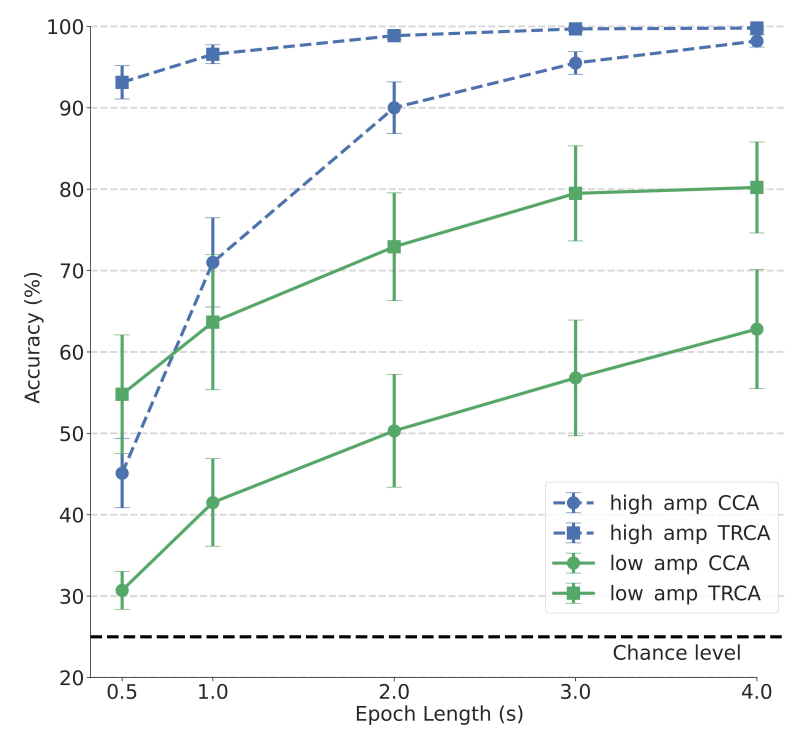

Fig. 4: Mean classification accuracy (with std. err.) over different data length $(0.5,1,2,34$ seconds) for high (red solid line) and low (blue dashed line) amplitude stimuli using TRCA (square markers) and CCA (circle markers) methods.

the frequency of repeated visual stimuli are two interesting and potentially complementary approaches to improve users experience of SSVEP-BCI. The group-level SNR analyses confirmed our intuition that the low amplitude stimuli could still elicit measurable brain responses. The topographical map revealed that these stimulations evoked typical neural activation which are maximal over occipital and parietooccipital cortices.

The classification performance of the low amplitude stimuli reached $80.2 \%$ of accuracy which is particularly promising considering the substantially reduced strength of the visual stimuli (one-tenth of standard amplitude). Beside satisfactory classification accuracy, the high amplitude stimuli required a longer data length (i.e. $3 s$ vs $0.5 s$ ). It brings here a genuine and open question about the overall comfort of a BCI with reduced amplitude stimuli. 
Is the increase of comfort with these low amplitude stimuli enough to counterbalance the frustration caused by longer stimulation and lower performances ? Nevertheless, it is worth noting that high classification accuracy was observed for some participants $(100 \%$ accuracy for P6 and P7). The wide range of classification performance for the low amplitude condition hints toward interindividual differences. It is however unclear whether these differences in classification accuracy are related to individual differences in terms of stimulus perception sensitivity or nonlinear interindividual differences in SSVEP responsivity to various degrees of stimulation intensity. Moving forward, a systematic investigation of individual perception thresholds and a gradation of SSVEP responses at different levels of amplitude modulation would shed light on this pending question. Moreover, there is a need for a definition of subjectspecific breaking points of such a low amplitude approach. This information could be used to inform the definition of optimal amplitude selection to strike the right balance between SSVEP signal strength and user comfort.

Eventually, our results demonstrate that the TRCA algorithm outperformed CCA for both low and high SSVEP. This result is in line with recent studies reporting better classification performance using TRCA [19] [4]. TRCA seems to be an effective approach to deal with the reduced signal strength of low amplitude RVS and should therefore be considered as a classification method moving forward. In contrast to TRCA, CCA does not require to be trained on prior data. Such a calibration-free approach is particularly desirable from a user-experience perspective as it reduces the set-up time of the BCI. Nevertheless, calibration duration can be optimized with more interactive and engaging protocols to reduce the perceived constraints. Further investigations into classification approaches applied to low amplitude stimuli will shed light on the range of solutions available.

\section{CONCLUSION AND PERSPECTIVES}

This study was dedicated to assessing the relevance of low amplitude flickers for BCI purposes. Our results, collected over 8 participants, revealed that low amplitude visual stimuli elicited typical SSVEP responses while improving participants' subjective visual comfort. The encouraging classification performance achieved using the TRCA classification method further demonstrate the validity of using low-intensity flickers within the frame of SSVEPBCI applications. Future work could extend the proposed approach to find the optimal compromise between global user experience and SSVEP signal strength following a subject-dependent and iterative approach. Moreover, the interaction between amplitude and other features of the flickering stimuli such as colour, duty cycle, patterns blur, or waveform shape may be studied to further reduce the intrusiveness of the visual stimuli. Eventually, the use of high flickering frequency could also present an alternative approach to promoting transparent and seamless brainmachine interaction for neuroergonomics applications [20].

\section{ACKNOWLEDGEMENT}

This work was funded by AID (Neurosynchrone project) and ANITI (Chair for Neuroadaptive Technology).

\section{REFERENCES}

[1] F. B. Vialatte, M. Maurice, J. Dauwels, and A. Cichocki, "Steady-state visually evoked potentials: Focus on essential paradigms and future perspectives," Progress in Neurobiology, vol. 90, no. 4, pp. 418-438, 2010.

[2] R. Srinivasan, F. A. Bibi, and P. L. Nunez, "Steady-state visual evoked potentials: Distributed local sources and wave-like dynamics are sensitive to flicker frequency," Brain Topography, 2006.

[3] C. S. Herrmann, "Human EEG responses to $1-100 \mathrm{~Hz}$ flicker: Resonance phenomena in visual cortex and their potential correlation to cognitive phenomena," Experimental Brain Research, 2001.

[4] M. Nakanishi, Y. Wang, X. Chen, Y. T. Wang, X. Gao, and T. P. Jung, "Enhancing detection of SSVEPs for a high-speed brain speller using task-related component analysis," IEEE Transactions on Biomedical Engineering, vol. 65, no. 1, pp. 104-112, 2018.

[5] Z. Lin, C. Zhang, W. Wu, and X. Gao, "Frequency recognition based on canonical correlation analysis for SSVEP-Based BCIs," IEEE Transactions on Biomedical Engineering, vol. 54, no. 6, pp. 11721176, 2007.

[6] T. Cao, F. Wan, C. M. Wong, J. N. da Cruz, and Y. Hu, "Objective evaluation of fatigue by EEG spectral analysis in steady-state visual evoked potential-based brain-computer interfaces," BioMedical Engineering Online, vol. 13, no. 1, pp. 1-13, 2014.

[7] A. M. Norcia, L. G. Appelbaum, J. M. Ales, B. R. Cottereaur, and B. Rossion, "The steady state VEP in research," Journal of Vision, vol. 15 , no. 6 , pp. 1-46, 2015.

[8] M. Labecki, R. Kus, A. Brzozowska, T. Stacewicz, B. S. Bhattacharya, and P. Suffczynski, "Nonlinear origin of SSVEP spectra-a combined experimental and modeling study," Frontiers in Computational Neuroscience, vol. 10, no. DEC, pp. 1-10, 2016.

[9] R. Kuś, A. Duszyk, P. Milanowski, M. Łabecki, M. Bierzyńska, Z. Radzikowska, M. Michalska, J. Zygierewicz, P. Suffczyński, and P. J. Durka, "On the Quantification of SSVEP Frequency Responses in Human EEG in Realistic BCI Conditions," PLoS ONE, vol. 8, no. 10, 2013.

[10] R. Ortner, B. Z. Allison, G. Korisek, H. Gaggl, and G. Pfurtscheller, "An SSVEP BCI to control a hand orthosis for persons with tetraplegia," IEEE Transactions on Neural Systems and Rehabilitation Engineering, vol. 19, no. 1, pp. 1-5, 2011.

[11] G. Rubboli, J. Parra, S. Seri, T. Takahashi, and P. Thomas, "EEG Diagnostic Procedures and Special Investigations in the Assessment of Photosensitivity," Epilepsia, vol. 45, no. SUPPL. 1, pp. 35-39, 2004.

[12] S. N. Ahmed, J. Unterman, B. McCloskey, and D. B. Sinclair, "Clinical significance of photic stimulation during routine EEGs of adult patients," Neurodiagnostic Journal, 2006.

[13] D. Kahneman, "Attention and Effort," The American Journal of Psychology, vol. 88, no. 2, p. 339, 1973.

[14] J. W. Peirce, "PsychoPy-Psychophysics software in Python," Journal of Neuroscience Methods, vol. 162, no. 1-2, pp. 8-13, 2007.

[15] M. X. Cohen and R. Gulbinaite, "Rhythmic entrainment source separation: Optimizing analyses of neural responses to rhythmic sensory stimulation," NeuroImage, vol. 147, no. November 2016, pp. 43-56, 2017.

[16] G. Bin, X. Gao, Z. Yan, B. Hong, and S. Gao, "An online multichannel SSVEP-based brain-computer interface using a canonical correlation analysis method," Journal of Neural Engineering, vol. 6, no. 4, 2009.

[17] X. Chen, Y. Wang, M. Nakanishi, X. Gao, T. P. Jung, and S. Gao, "High-speed spelling with a noninvasive brain-computer interface," Proceedings of the National Academy of Sciences of the United States of America, vol. 112, no. 44, pp. E6058-E6067, 2015.

[18] H. Tanaka, T. Katura, and H. Sato, "Task-related component analysis for functional neuroimaging and application to near-infrared spectroscopy data," NeuroImage, vol. 64, pp. 308-327, 2013.

[19] X. Xing, Y. Wang, W. Pei, X. Guo, Z. Liu, F. Wang, G. Ming, H. Zhao, Q. Gui, and H. Chen, "A high-speed SSVEP-based BCI using dry EEG electrodes," Scientific Reports, vol. 8, no. 1, pp. 1-10, 2018.

[20] F. Dehais, A. Lafont, R. Roy, and S. Fairclough, "A Neuroergonomics approach to mental workload, engagement and human performance," Frontiers in neuroscience, vol. 14, p. 268, 2020. 